\title{
Quantum and classical correlations in waveguide lattices
}

\author{
Yaron Bromberg, ${ }^{1}$ Yoav Lahini, ${ }^{1}$ Roberto Morandotti, ${ }^{2}$ and Yaron Silberberg, ${ }^{1}$, \\ ${ }^{1}$ Department of Physics of Complex Systems, Weizmann Institute of Science, Rehovot 76100, Israel \\ ${ }^{2}$ Institute National de la Recherche Scientifique, Varennes, Qúbec, Canada
}

(Dated: March 31, 2009)

\begin{abstract}
We study quantum and classical Hanbury Brown-Twiss correlations in waveguide lattices. We develop a theory for the propagation of photon pairs in the lattice, predicting the emergence of nontrivial quantum interferences unique to lattice systems. Experimentally, we observe the classical counterpart of these interferences using intensity correlation measurements. We discuss the correspondence between the classical and quantum correlations, and consider path-entangled input states which do not have a classical analogue. Our results demonstrate that waveguide lattices can be used as a robust and highly controllable tool for manipulating quantum states, and offer new ways of studying the quantum properties of light.
\end{abstract}

Lattices of coupled waveguides have been shown to be extremely versatile in manipulating the flow of light [1, 2]. Such systems enabled direct observation of optical analogues of many fundamental quantum mechanical effects such as Bloch oscillations [3, 4], Anderson localization [5, 6, 7], quantum Zeno effect [8], quantum walks [9] and many others [1, 2, 10]. However, these effects were all related to the wave properties of light rather than to its particle nature. To observe the quantum properties of light, one should consider correlations between single photons. Here we show that photon pairs propagating in waveguide lattices develop nontrivial quantum correlations unique to lattice systems. We experimentally observe some of these features, albeit with reduced contrast, using Hanbury Brown-Twiss intensity correlation measurements with phase-averaged coherent states. Our results suggest that waveguide lattices can be used as a robust and highly controllable tool for manipulating quantum states in an integrated manner, offering new ways of studying quantum properties of light in structured media.

Consider a lattice that is made of identical optical waveguides, each supporting a single transverse mode, as shown schematically in Fig. 1a. The evolution of the quantized electromagnetic field in each waveguide is given by the Heisenberg equation for the bosonic creation operator $a^{\dagger}$. For a lattice with nearest-neighbors couplings, the Heisenberg equation for the creation operator of the $k^{t h}$ waveguide is given by:

$$
i \frac{n}{c} \frac{\partial a_{k}^{\dagger}}{\partial t}=i \frac{\partial a_{k}^{\dagger}}{\partial z}=\beta a_{k}^{\dagger}+C_{k, k+1} a_{k+1}^{\dagger}+C_{k, k-1} a_{k-1}^{\dagger},
$$

where $z$ is the spatial coordinate along the propagations axis, $\beta$ is the propagation constant of the waveguides, $C_{k, k \pm 1}$ are the coupling constants between adjacent waveguides, and $c / n$ is the speed of light in the medium. The creation operators at any point along the propagation are calculated by integrating Eq. (1):

$$
a_{k}^{\dagger}(z)=e^{i \beta z} \sum_{l} U_{k, l}(z) a_{l}^{\dagger}(z=0), U_{k, l}(z)=\left(e^{i \beta z C}\right)_{k, l} .
$$

$U_{k, l}(z)$ is a unitary transformation given by calculating the exponent of the coupling matrix $i z C_{k, l}$, which describes the amplitude for the transition of a single photon from waveguide $l$ to waveguide $k$. Since any input state can be expressed with the creation operators $a_{l}^{\dagger}$ and the vacuum state $|0\rangle$, the evolution of non classical states along the lattice can be calculated using Eq. (2). When a single photon is coupled to waveguide $l$, the input state $a_{l}^{\dagger}|0\rangle \equiv|1\rangle_{l}$ will evolve to the superposition $\sum_{k} U_{l, k}^{*}|1\rangle_{k}$, where $|1\rangle_{k}$ is the state of a single photon occupying waveguide $k$. However, measurements of the probability distribution of single photons are not enough to reveal the quantum properties of light, as the probability distribution of a single photon $\left|U_{k, l}(z)\right|^{2}$ evolves in the same way as the intensity distribution of classical light $[9,12,13$. The quantum mechanical properties of light are observed when correlations between the propagating photons are considered. In this Letter we focus on the evolution of the photon-number correlation function $\Gamma_{q, r}=\left\langle a_{q}^{\dagger} a_{r}^{\dagger} a_{r} a_{q}\right\rangle$, when two indistinguishable photons are injected into the lattice.

We start by analyzing the simplest example of two coupled waveguides. The coupling matrix in this case is $\overleftrightarrow{C}=\left(\begin{array}{cc}0 & C \\ C & 0\end{array}\right)$, and the transformation $U_{k, l}(z)$ is therefore $\overleftrightarrow{U}(z)=\left(\begin{array}{cc}\cos (C z) & i \sin (C z) \\ i \sin (C z) & \cos (C z)\end{array}\right)$. The coupler acts as a beam splitter, with the reflection and transmission coefficients varying continuously along the propagation. If two photons are injected to the coupler, one to each waveguide, the average photon number at each of the waveguides is constant since $n_{1(2)}(z)=$ $\left\langle a_{1(2)}^{\dagger} a_{1(2)}\right\rangle=\left|U_{11}\right|^{2}+\left|U_{12}\right|^{2}=1$. The nonclassical nature of the light is revealed by considering $\Gamma_{1,2}$, the probability to detect exactly one photon at each waveguide (a coincidence measurement). Using Eq. (2) we obtain $\Gamma_{1,2}(z)=\left|U_{11} U_{22}+U_{12} U_{21}\right|^{2}=\operatorname{Cos}^{2}(2 C z)$. Since two paths lead to the final state of one photon at each waveguide, they interfere and the probability for a coincidence measurement oscillates along the propagation. After 

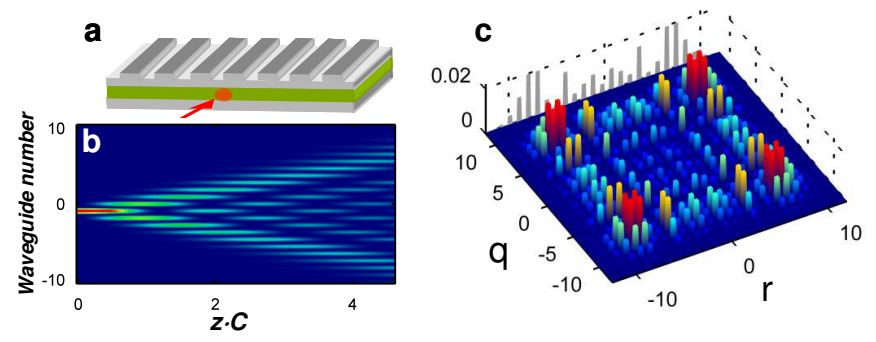

Figure 1: (a) A schematic view of the waveguide lattice used in the experiment. The red arrow represents the input light beam. (b) The calculated probability distribution $\left\langle n_{q}(z)\right\rangle$ of a single photon injected to the central waveguide of a periodic lattice, as a function of the propagation distance. The photon couples coherently from each waveguide to its neighbors, and the probability distribution concentrates at two outer lobes. (c) The calculated correlation matrices $\Gamma_{q, r}$, representing the probability to detect at the output of the lattice exactly one photon at waveguide $r$ and one photon at waveguide $q$, when both photons are coupled to a single waveguide at the center of the lattice, i.e. $\left|\varphi_{0}\right\rangle \equiv 1 / \sqrt{2} a_{0}^{\dagger 2}|0\rangle$. This matrix is a simple product of two single-photon distributions, thus showing no quantum interference. The grey bars are obtained by summing the matrix along one axis, representing the results of a single photon measurement.

propagating exactly half a coupling length $z_{B S}=\pi / 4 C$, we find $\Gamma_{1,2}\left(z_{B S}\right)=0$. At this point the two photons bunch, and are found together in either one of the two waveguides. $\overleftrightarrow{U}\left(z_{B S}\right)$ is identical to the transformation of a symmetric beam splitter, and the coincidence measurement vanishes in the same manner as in the HongOu-Mandel (HOM) interferometer [14]. Since a pair of coupled waveguides is equivalent to a beam splitter, it is possible to cascade several of them in order to implement quantum gates in an integrated manner [15]. A lattice of many coupled waveguides enriches the variety of correlations obtained in integrated structures, as we show bellow.

We now turn to study the quantum properties of a periodic lattice with a large number of identical waveguides, where all the coupling constants are equal $C_{n, n \pm 1}=C$. As long as the photons are far from the boundaries of the lattice, Eq. (2) yields $U_{q, k}(z)=i^{q-k} J_{q-k}(2 C z)$, where $J_{q}$ is the $q^{\text {th }}$ Bessel function [2, 16]. When a single photon is coupled to waveguide $k$, it will evolve to waveguide $q$ with a probability $n_{q}=\left|U_{q, k}(z)\right|^{2}=J_{q-k}(2 C z)^{2}$. The photon spreads across the lattice by coupling from one waveguide to its neighbors in a pattern characterized by two strong 'ballistic' lobes, as shown in Fig. 1b. If a second photon is coupled to another waveguide $l$, then the average photon number at waveguide $q$ is simply the incoherent sum $n_{q}=<a_{q}^{\dagger} a_{q}>=J_{q-k}(2 C z)^{2}+J_{q-l}(2 C z)^{2}$. Once again, the quantum nature of light is revealed by considering the correlations between the two photons. In the following we study the correlation $\Gamma_{q, r}$ for three distinct two-photon input states: i) Both photons are cou-
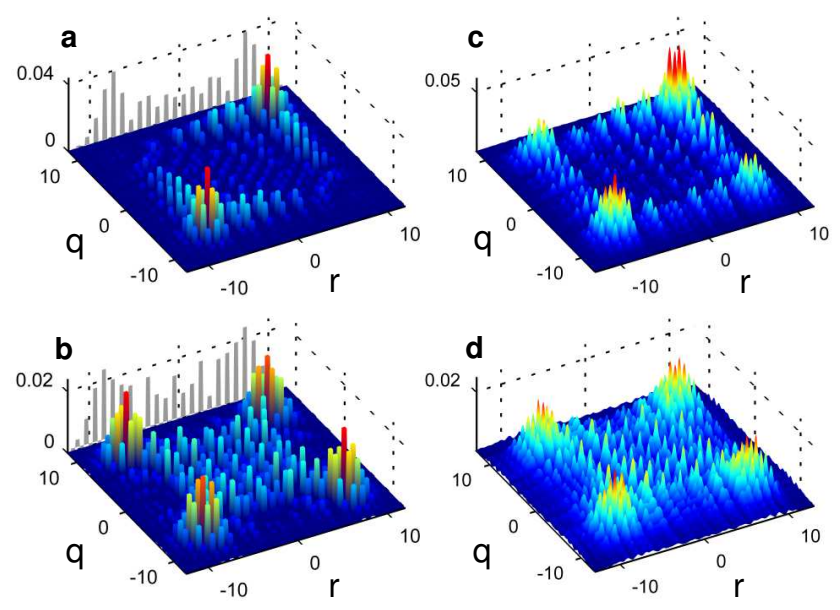

Figure 2: Quantum and classical correlations in waveguide lattices. (a) The correlation matrix $\Gamma_{q, r}$ when the photons are coupled to two adjacent waveguides i.e. $\left|\varphi_{1}\right\rangle \equiv a_{0}^{\dagger} a_{1}^{\dagger}|0\rangle$. The two photons exhibit bunching, and will emerge from the same side of the lattice. (b) The correlation matrix when the two photons are coupled to two waveguides separated by one waveguide, $\left|\varphi_{2}\right\rangle \equiv a_{-1}^{\dagger} a_{1}^{\dagger}|0\rangle$. Here the two photons will emerge either both from the lobes, or both from the center. (c) Measured classical intensity correlations $\Gamma_{q, r}^{(c)}$, corresponding to the $\left|\varphi_{1}\right\rangle$ input state. (d) Measured classical intensity correlations $\Gamma_{q, r}^{(c)}$, corresponding to the $\left|\varphi_{2}\right\rangle$ input state.

pled to a single waveguide at the center of the lattice, i.e. $\left|\varphi_{0}\right\rangle \equiv 1 / \sqrt{2} a_{0}^{\dagger 2}|0\rangle$ (ii) The two photons are coupled to two adjacent waveguides $\left|\varphi_{1}\right\rangle \equiv a_{0}^{\dagger} a_{1}^{\dagger}|0\rangle$ and iii) The two photons are coupled to two waveguides, separated by one waveguide, $\left|\varphi_{2}\right\rangle \equiv a_{-1}^{\dagger} a_{1}^{\dagger}|0\rangle$. The correlation matrix $\Gamma_{q, r}$ represents the probability to detect one photon at waveguide $q$ and its twin photon at waveguide $r \neq q$. The probability to detect both photons at the same waveguide $q$ is given by $\Gamma_{q, q} / 2$.

Fig. 1c depicts the correlation matrix $\Gamma_{q, r}$ at the output of the lattice, when the two photons are coupled to the same input waveguide (the $\left|\varphi_{0}\right\rangle$ input state). In this case, there is no interference and the correlation matrix is just a product of the two classical probability distributions, $\Gamma_{q, r}=2\left|U_{q 0} U_{r 0}\right|^{2}$. The correlation map is characterized by four strong lobes at the corners of the matrix, resulting from the tendency of the photons to propagate in the ballistic directions.

When the two photons are coupled to two neighboring sites, i.e. the $\left|\varphi_{1}\right\rangle$ state, the correlation map changes considerably as shown in Fig. 2a. The most obvious feature is the vanishing of the two 'off-diagonal' lobes: the photons tend to bunch to the same lobe. This can be thought of as a generalized HOM interference. Two paths lead to a coincidence measurement between waveguide $q$ and waveguide $r$ : either the photon from waveguide 0 propagates to waveguide $q$ and the photon from waveguide 1 to waveguide $r$, or vice versa - from waveguide 
0 to waveguide $r$ and from waveguide 1 to waveguide $q$ [17]. These paths are complex and involve hopping of the photons between many waveguides, nevertheless they interfere and the correlation matrix is thus given by $\Gamma_{q, r}=\left|U_{q 0} U_{r 1}+U_{q 1} U_{r 0}\right|^{2}$. The destructive interference which leads to vanishing of the off-diagonal lobes can be mathematically traced to the inherent $90^{\circ}$ phase shift associated with nearest-neighbors coupling. We note that since the photons tunnel between the waveguides continuously, the visibility of the quantum interference only weakly depends on the overall length of the lattice.

The four lobes are recovered when the photons are initiated in the $\left|\varphi_{2}\right\rangle$ state, i.e. with one waveguide separation between the input sites (Fig 2b). However, this state also contains strong non-classical features - note the differences between Fig. 1c and 2b. The photon pair exhibit bunching but with a different symmetry: if one photon is detected in between the lobes, the probability to detect the second photon in a lobe vanishes, even though a single photon is most likely to reach the lobes. Similarly, if one photon is detected in a lobe, it is certain that the other photon is also in a lobe. It is important to note that in the above examples, the quantum interference emerges since the two photons are indistinguishable. Interferences with distinguishable particles are possible, but require an entangled input state [18].

Some of the special features of these quantum mechanical correlations can be captured using intensity correlation measurements with classical light [19, 20, 21]. The quantum mechanical probability to detect one photon at waveguide $q$ and its twin photon at waveguide $r$ is related to the classical intensity correlation function $\Gamma_{q, r}^{(c)}=\left\langle I_{q} I_{r}\right\rangle$ where $\langle\cdot\rangle$ denotes statistical (or temporal) averaging. This non-local intensity correlation function is usually discussed in the context of the classical Hanbury Brown-Twiss effect and its quantum interpretation [11, 22]. We studied experimentally intensity correlations at the output of a periodic waveguide lattice. The lattice of 89 identical waveguides was fabricated on an AlGaAs substrate using e-beam lithography, followed by reactive ion etching. Each waveguide is $8 \mathrm{~mm}$ long and $4 \mu \mathrm{m}$ wide [23]. The tunneling parameter between sites $C$ is determined by the etch depth of the sample $(1.3 \mu \mathrm{m})$ and by the distance between neighboring waveguides $(4 \mu \mathrm{m})$ and was measured to be $290 \mathrm{~m}^{-1}$. We used an OPO (Spectra-Physics, OPAL) pumped by a modelocked Ti:Sapphire laser (Spectra-Physics Tsunami) to generate $150 \mathrm{fs}$ pulses, at a wavelength of $1530 \mathrm{~nm}$ with $80 \mathrm{MHz}$ repetition rate. The average power was on the order of $0.1 \mathrm{~mW}$, thus nonlinear effects were negligible.

Two-photon input states can be mimicked by injecting into the lattice two phase-averaged coherent states: two coherent states with the same mean photon number and a fluctuating relative phase [19, 25]. Thus, we injected two coherent beams into two different sites of the lattice [24], and randomized their relative phase with
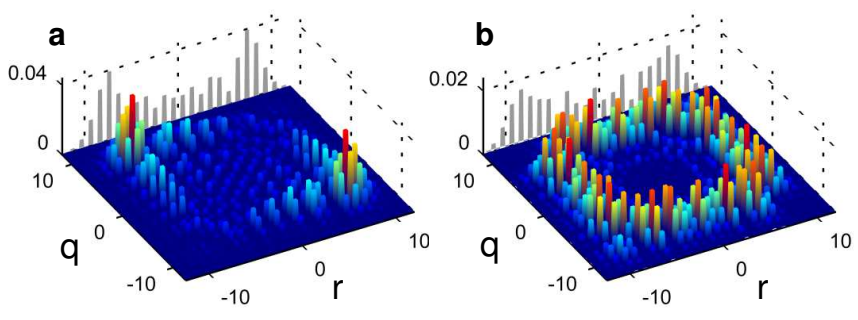

Figure 3: Quantum correlation maps $\Gamma_{q, r}$ for path-entangled input states. (a) The two photons are injected together to either of two neighboring waveguides, $\left|\psi^{(+)}\right\rangle=$ $\frac{1}{2}\left(a_{1}^{\dagger 2}+a_{0}^{\dagger 2}\right)|0\rangle$. The correlation is significant only in the 'off-diagonal' peaks, which indicates that the two photons will emerge from opposite sides of the lattice. (b) The correlation map for an input state with two photons in either of two waveguides with one waveguide separation, where there is a $\pi$-phase between the two possibilities, $\left|\psi^{(-)}\right\rangle=$ $\frac{1}{2}\left(a_{1}^{\dagger 2}-a_{-1}^{\dagger 2}\right)|0\rangle$.

a spatial light modulator. For each phase realization, the intensity profile at the output facet of the slab was imaged on an infrared camera, and the intensity correlations between all the waveguides were computed. The intensity-correlation function $\Gamma_{q, r}^{(c)}$ was obtained by averaging the measured correlations over many phase realizations. The measured intensity correlations for the nearest neighbors (mimicking the $\left|\varphi_{1}\right\rangle$ state) and next-nearest neighbors $\left(\left|\varphi_{2}\right\rangle\right.$ state) inputs are presented in figures $2 \mathrm{c}$ and $2 \mathrm{~d}$, correspondingly. The patterns are strikingly similar to the corresponding quantum correlations $\Gamma_{q, r}$, except for the reduced contrast. Indeed, for two incoherent sources coupled to waveguides $k$ and $l \neq k$, the classical correlation is given by $\Gamma_{q, r}^{(c)}(z)=$ $I_{0}^{2}\left(\left|U_{q l} U_{r k}+U_{q k} U_{r l}\right|^{2}+\left|U_{q l} U_{r l}\right|^{2}+\left|U_{q k} U_{r k}\right|^{2}\right)$, where $I_{0}$ is the intensity coupled to each waveguide. The last two terms are responsible for the reduced contrast. Thus, while the 'off-diagonal' lobes practically vanish for the quantum input state $\left|\varphi_{1}\right\rangle$, a straightforward calculation shows that for classical light $\Gamma_{q, r}^{(c)}>\frac{1}{3} \sqrt{\Gamma_{q, q}^{(c)} \Gamma_{r, r}^{(c)}}$, in agreement with our experimental results. Still these classical Hanbury Brown-Twiss type intensity correlations do echo many of the special features of the quantum correlations of Fig. 2a,b.

The great potential of waveguide lattices for quantum information probably lies in the extension of these concepts to non-uniform lattices in order to specially design the correlation properties, and in the utilization of these multiport systems for handling more complex quantum states. As an example for the latter, consider the propagation of a path-entangled input state with two photons in either of two neighboring waveguides, $\left|\psi^{(+)}\right\rangle=\frac{1}{2}\left(a_{1}^{\dagger 2}+a_{0}^{\dagger 2}\right)|0\rangle$. The calculated correlation map for this case is presented in Fig 3a. The corre- 
lation in the 'diagonal' peaks completely vanishes and is significant only in the 'off-diagonal' peaks. Accordingly, the two photons will always separate and emerge from different sides of the lattice. The corresponding correlation map violates the Cauchy-Schwarz inequality $\Gamma_{q, r}<\sqrt{\Gamma_{q, q} \Gamma_{r, r}}$, indicating that this case has no apparent classical analog. As another illustration, consider the state $\left|\psi^{(-)}\right\rangle=\frac{1}{2}\left(a_{1}^{\dagger 2}-a_{-1}^{\dagger 2}\right)|0\rangle$. Now the photons are in either of two next-nearest neighboring waveguides, yet with a $\pi$-phase shift. The correlation map shown in Fig. $3 \mathrm{~b}$ reveals that in this case, one photon will always reach a lobe while the other will always reach the center.

In this Letter we studied the evolution of photon pairs along periodic lattices, and have shown that the resulting quantum correlations strongly depend on the input states. We compared our predictions with a classical wave theory, and experimentally demonstrated that some features can be obtained using classical intensity correlation measurements, yet with reduced contrast. The correspondence between the classical and quantum nature of light can be further studied by considering the evolution of quantum correlations in the presence of dephasing, which can be introduced via lattice inhomogeneities. Furthermore, waveguide lattices offer new possibilities as they can be designed in ways that are not feasible using bulk or fiber optical systems. It will be especially interesting to study the effect of such lattices on the propagation of other types of non-classical light, such as squeezed states [26] and cat states.

We would like to thank H. S. Eisenberg, N. Bar-Gill and H. B. Perets for valuable help. Financial support by NSERC and CIPI (Canada), and EPRSC (UK) is gratefully acknowledged. YL is supported by the Adams Fellowship of the Israel Academy of Sciences and Humanities.

\footnotetext{
* yaron.silberberg@weizmann.ac.il
}

[1] D. N. Christodoulides, F. Lederer, and Y. Silberberg, Nature 424, 817 (2003).

[2] F. Lederer, G. I. Stegeman, D. N. Christodoulides, G. Assanto, M. Segev, and Y. Silberberg, Phys. Rep. 463, 1-126 (2008).

[3] R. Morandotti, U. Peschel, J. S. Aitchison, H. S. Eisenberg, and Y. Silberberg, Phys. Rev. Lett. 83, 4756-4759
(1999).

[4] T. Pertsch, P. Dannberg, W. Elflein, A. Brauer, and F. Lederer, Phys. Rev. Lett. 83, 4752-4755 (1999).

[5] T. Pertsch, U. Peschel, J. Kobelke, K. Schuster, H. Bartelt, S. Nolte, A. Tunnermann, and F. Lederer, Phys. Rev. Lett. 93, 053901 (2004).

[6] T. Schwartz, G. Bartal, S. Fishman, and M. Segev, Nature 446, 52-55 (2007).

[7] Y. Lahini, A. Avidan, F. Pozzi, M. Sorel, R. Morandotti, D.N. Christodoulides, and Y. Silberberg, Phys. Rev. Lett. 100, 013906 (2008).

[8] P. Biagioni, G. Della Valle, M. Ornigotti, M. Finazzi, L. Duò, P. Laporta, and S. Longhi, Opt. Express. 16 3762-7 (2008).

[9] H. B. Perets, Y. Lahini, F. Pozzi, M. Sorel, R. Morandotti, and Y. Silberberg, Phys. Rev. Lett. 100, 170506 (2008).

[10] H. Trompeter, T. Pertsch, F. Lederer, D. Michaelis, U. Streppel, A. Brauer, and U. Peschel, Phys. Rev. Lett. 96, 023901 (2006).

[11] R. Hanbury Brown, and R. Q. Twiss, Nature 177, 27 29 (1956).

[12] H. Jeong, M. Paternostro, and M. S. Kim, Phys. Rev. A 69, 012310 (2004).

[13] P. K. Pathak, and G. S. Agarwal, Phys. Rev. A 75 , 032351 (2007).

[14] C. K. Hong, Z. Y. Ou, and L. Mandel, Phys. Rev. Lett. 59, 2044-2046 (1987).

[15] A. Politi, M. J. Cryan, J. G. Rarity, S. Yu, and J. L. O'Brien, Science 320, 646 - 649 (2008).

[16] A. Yariv, Quantum Electronics Ch. 22 (Wiley, New-York, 1989).

[17] K. Mattle, M. Micher, H. Weinfurter, A. Zeilinger, and M. Zukowski, Appl. Phys. B 60, S111-S117 (1995).

[18] Y. Omar, N. Paunkovic, L. Sheridan, and S. Bose, Phys. Rev. A 74, 042304 (2006).

[19] H. Paul, Rev. Mod. Phys. 58, 209-231 (1986).

[20] R. S. Bennink, S. J. Bentley, R. W. Boyd, and J. C. Howell, Phys. Rev. Lett. 92, 033601 (2004).

[21] K. J. Resch, P. Puvanathasan, J. S. Lundeen, M. W. Mitchell, and K. Bizheva, Opt. Exp. 15 8797-8804.

[22] R. J. Glauber, Phys. Rev. Lett. 10, 84-86 (1963).

[23] H. S. Eisenberg, Y. Silberberg, R. Morandotti, A. R. Boyd, and J. S. Aitchison, Phys. Rev. Lett. 81, 3383 (1998).

[24] A. Szameit, F. Dreisow, H. Hartung, S. Nolte, A. Tunnermann, and F. Lederer, Appl. Phys. Lett. 90, 241113 (2007).

[25] L. Mandel, Phys. Rev. A 28, 929-943 (1983).

[26] A. Rai, G. S. Agarwal, and J. H. H. Perk, Phys. Rev. A 78, 042304 (2008). 